Hales, PW; Olsen, ØE; Sebire, NJ; Pritchard-Jones, K; Clark, CA; (2015) A multi-Gaussian model for apparent diffusion coefficient histogram analysis of Wilms' tumour subtype and response to chemotherapy. NMR Biomed 10.1002/nbm.3337.

\title{
Article
}

\section{A multi-Gaussian model for apparent diffusion coefficient histogram analysis of Wilms' tumour subtype and response to chemotherapy}

Patrick W. Hales ${ }^{1 *}$, Oystein E. Olsen ${ }^{2}$, Neil J. Sebire ${ }^{3}$, Kathy Pritchard-Jones ${ }^{3}$ and Chris A. Clark $^{1}$

${ }^{1}$ Developmental Imaging and Biophysics Section, Institute of Child Health, University College London, London, WC1N 1EH, UK.

${ }^{3}$ Developmental Biology \& Cancer, Institute of Child Health, University College London, London, WC1N 1EH, UK.

${ }^{2}$ Radiology Department, Great Ormond Street Hospital, London, WC1N 3JH, UK

* Correspondence to: Patrick Hales, Developmental Imaging and Biophysics Section, University College London Institute of Child Health, 30 Guilford Street, London, WC1N 1EH, UK. Tel: +44 (0)20 7905 2129. Fax: +44 (0)20 7905 2358. Email: p.hales@ucl.ac.uk. Financial support provided by Cancer Research UK, Grant Number C7809/A10342 and Great Ormond Street Hospital Children's Charity.

\section{Word count:}

5377

Keywords:

Wilms' tumour, DWI, ADC, histogram analysis.

\section{Abbreviations:}

DWI diffusion-weighted imaging, WT Wilms' tumour, ADC apparent diffusion coefficient, CT computed tomography, ANOVA Analysis of variance, ROC receiver operator characteristic, SIOP Society of Pediatric Oncology. 


\begin{abstract}
Wilms' tumours (WT) are large heterogeneous tumours, which typically consist of a mixture of histological cell types, along with regions of chemotherapy induced regressive change and necrosis. The predominant cell type in a WT is assessed histologically following nephrectomy, and used to assess the tumour subtype and potential risk. The purpose of this study was to develop a mathematical model to identify sub-regions within WTs with distinct cellular environments in vivo, determined using Apparent Diffusion Coefficient (ADC) values from diffusion-weighted imaging (DWI). We recorded WT subtype from the histopathology of 32 tumours resected in patients who received DWI prior to surgery, after pre-operative chemotherapy had been administered. In 23 of these tumours, DWI data were also available prior to chemotherapy. Histograms of ADC values were analysed using a multi-Gaussian model fitting procedure, which identified 'sub-populations' with distinct cellular environments within the tumour volume. The mean and lower quartile ADC values of the predominant viable-tissue sub-population $\left(A D C_{1 M E A N}, A D C_{1 L Q}\right)$, along with the same parameters from the entire tumour volume $\left(A D C_{O M E A N}, A D C_{0 L Q}\right)$, were tested as predictors of WT subtype. $A D C_{1 L Q}$ from the multi-Gaussian model was the most effective parameter for stratification of WT subtype, with significantly lower values observed in high-risk blastemal-type WTs compared to intermediate risk stromal, regressive and mixed-type $(p<0.05)$. No significant difference in $A D C_{1 L Q}$ was found between blastemal-type WTs and intermediate risk epithelial-type. The predominant viable tissue sub-population in every stromal-type WT underwent a positive shift in $A_{1} C_{1 M E A N}$ after chemotherapy. Our results suggest that our multi-Gaussian model is a useful tool for differentiating distinct cellular regions within WTs, which helps to identify the predominant histological cell type in the tumour in vivo. This shows potential for improving the risk-based stratification of patients at an early stage, and for guiding biopsies to target the most malignant part of the tumour.
\end{abstract}

\title{
Introduction
}

Malignant renal tumours comprise $6 \%$ of all childhood cancers. Wilms' tumour (WT), or nephroblastoma, is by far the most frequent type, accounting for over $90 \%$ of renal tumours, of which approximately $7 \%$ are bilateral [1]. In Europe, WT is treated with pre-operative chemotherapy, followed by surgery. The aim of chemotherapy is to reduce the tumour size to facilitate subsequent surgery, decrease the risk of intra-operative tumour rupture, and to downstage the tumour to reduce post-operative chemotherapy. Radiological assessment of chemotherapy response has traditionally been measured using change in tumour volume on imaging. However, this can be unreliable in the presence of substantial necrosis, and tissue differentiation may occur despite an increase in volume [2]. Furthermore, tumours which shrink in volume may still be composed predominantly of malignant cells [3], [4].

After chemotherapy, a full or partial nephrectomy is performed and the excised tumour is classified histologically. Traditionally, WT are divided into two groups: favourable or anaplastic histology, with the latter demonstrating nuclear enlargement, nuclear atypia, and irregular mitotic figures. The frequency of anaplasia is about $5 \%$, and these patients are considered particularly high-risk, with an estimated recurrence free survival rate of $40-50 \%$ [5], [6]. WTs are further classified according to the predominant features of the tissue after chemotherapy. If $>66 \%$ of the tissue has undergone chemotherapy-induced change, the tumour is classified as regressive, or completely necrotic if no viable tumour tissue remains. If $<66 \%$ of the tumour shows chemotherapy induced change, and one of stromal, epithelial or blastemal cells represent $>66 \%$ of the viable tissue, the tumour is classified according to the predominant cellular subtype; otherwise, the tumour is classified as mixed type [7]. 
Patients with epithelial, stromal, mixed and regressive WTs generally have very favourable outcomes (>89\% recurrence free survival after 4 years [8]), and are classified according to the International Society of Pediatric Oncology (SIOP) protocol as intermediate risk [7], [9]. Patients with blastemal WTs have a less favourable outcome $(62 \%$ recurrence free survival rate after 5 years [10]), and are classified as high-risk.

Although the overall survival rate in most WT subtypes is very high, patients are vulnerable to the late effects of treatment, with nearly one quarter reporting a significant chronic health condition by 25 years of follow up [11]. As such, the focus of management in this disease has shifted to improving risk-based stratification, to maintain excellent outcomes in low risk patients while decreasing the toxicity and burden of treatment. Conversely, it is important to identify patients with high risk tumours early, as these may benefit from intensified therapy [12]. This would require early identification of WT subtype, and a good understanding of how each subtype responds to chemotherapy. However, at present WT subtype can only be determined by histological assessment of the tumour tissue, after pre-operative chemotherapy and nephrectomy. Given the heterogeneous nature of WTs, needle biopsies are susceptible to sampling errors [13], and therefore non-invasive imaging techniques have a vital role to play in both early patient risk-stratification and guiding biopsies to focus on the most malignant part of the tumour.

Although Computed tomography (CT) is often used for imaging and staging of WT, magnetic resonance imaging (MRI) offers superior spatial resolution and tissue contrast, does not use ionizing radiation, and is therefore the preferred imaging modality. Diffusion-weighted imaging (DWI) is an MRI technique which measures the apparent diffusion coefficient (ADC) of water molecules in the tissue, which acts as a surrogate biomarker of cellular density [14]. Advances in MR imaging technology in recent years have led to increasing use of DWI in extra-cranial imaging applications, and ADC values have been shown to be useful in characterising tumours in the breast [15], liver [16], prostate [17], and kidney [18], [19]. However, the use of DWI in WT is relatively unexplored. Humphries et al. reported a negative correlation between $A D C$ and histological features (cell density) in a range of extracranial mass lesions (three of which were WTs) [14]. McDonald et al. examined patterns of shift in ADC histograms in abdominal tumours during chemotherapy (six of which were WTs), however, due to the small cohort used in this study, no attempt was made to differentiate WT subtypes [2]. Furthermore, it is common for the mean ADC value within a tumour to be reported, however, as WTs are heterogeneous entities, a mixture of cellular environments can be present within a single WT, in which case a single mean is not representative of the underlying tissue. An approach taken by others is to define ROls in homogenous regions of the tumour, often only in a single slice - however, this approach can be subjective, and does not cover the entire tumour volume, and is therefore prone to sampling errors. In this work we developed a mathematical model which identifies 'subpopulations' in histograms of ADC values which represent distinct cellular environments, throughout the entire tumour volume. We aimed to use this model to: (a) distinguish viable and non-viable tumour tissue, (b) identify the most prominent viable tissue sub-population, and (c) explore the link between the ADC values in this sub-population with histological assessment of the tumour. 


\section{Materials and Methods}

\section{Patients}

Data were collected retrospectively from routine healthcare records of children who were referred for MRI assessment of primary abdominal solid tumours at our institution between January 2006 and October 2013. Local institutional research office approval was granted for this retrospective case note and imaging review. Only children diagnosed with WT, who received DWI prior to nephrectomy (following pre-operative chemotherapy) were included in this study. Exclusion criteria were tumours where the largest area on a single imaging slice was $<3 \mathrm{~cm}^{2}$, and where specimens were unavailable for histopathological assessment. A total of 26 patients met the inclusion criteria for this study, 6 of whom had independently histologically-classified bilateral tumours, bringing the total number of tumours to 32 . In 23 of these tumours, DWI data were also acquired prior to chemotherapy. Children were awake, sedated or under general anaesthesia during the scan, depending on their age. Histological sections from surgically resected tumours were examined and reported according to current SIOP practice guidelines, in which tumours were classified according to the predominant histological cell type in the tumour tissue.

\section{MRI}

All scans were performed on a $1.5 \mathrm{~T}$ Siemens Magnetom Avanto scanner (Siemens, Erlangen, Germany), equipped with $40 \mathrm{mT} / \mathrm{m}$ gradients. A suitably sized multichannel body coil was chosen based on patient size, with a preference for the smallest coil combination that covered the whole abdomen and pelvis.

Conventional imaging sequences were acquired in each patient according to our institution's clinical protocol, which included coronal and axial 2D dual-echo short-tau inversion recovery (STIR), Axial T2-weighted fast spin-echo, and fat-suppressed T1w images before and after intravenous administration of gadolinium-based contrast (details in [2]). In addition, diffusionsensitised 2D single-shot echo-planar imaging was performed in the axial plane during free breathing. This was planned using the conventional sequences, by centering the acquisition slab on the tumour and using up to 19 sections to cover the entire tumour volume. Scan parameters were: slice thickness, $6 \mathrm{~mm}$; intersection gap, 1.2-2.4 mm, TR/TE, 2,600 ms/89 $\mathrm{ms}$; field of view, $350 \mathrm{~mm}$ with a rectangular factor of $75 \%$; echo-planar readout with matrix of 128 and 96 phase-encoding steps; receive bandwidth, 1,500 Hz/pixel; parallel imaging factor 2. We used signal averaging of six acquisitions and diffusion-sensitising gradients in three orthogonal planes with b-values of 0,500 and $1,000 \mathrm{~s} / \mathrm{mm}^{2}$. Fat signal was suppressed using a frequency-selective radio-frequency preparation pulse. Total acquisition time for this sequence was $5.3 \mathrm{~min}$, and diffusion-weighted images were always acquired prior to administration of gadolinium-based contrast material.

\section{Post Processing}

All data analysis was performed using Matlab R2014a (MathWorks Inc., Natick, MA). ADC maps were collected in each patient, which were produced by calculation of the slope of the natural logarithm of mean signal intensity (over three orthogonal planes) vs. b-value. Regions of interest (ROIs) were drawn around the perimeter of each tumour on every $b_{0}$ (non-diffusion weighted) imaging slice, provided the tumour area was greater than $3 \mathrm{~cm}^{2}$, to avoid partial volume artefacts. All ROls were either drawn or checked by a consultant radiologist with 12 years experience in paediatric radiology. Conventional MR images acquired during the same scan session were used to guide tracing of the tumour outline. 
T1w images acquired both pre- and post- gadolinium contrast agent injection were not available in all patients, and in some others these images were rejected when they were severely degraded by respiratory motion artefacts; overall 15 patients had good quality preand post-gadolinium T1w images. In these patients, a further 'viable tumour' mask was created as follows: firstly, signal intensity from pre-gadolinium T1w images was subtracted from post-gadolinium T1w images. The subtracted set was then thresholded to include only voxels with a value above that of the erector spinae muscles (similar to [2]) - voxels with signal intensity below this threshold were classified as non-enhancing / necrotic regions (i.e. regions in which the uptake of gadolinium is minimal). Medlnria software (Inria, Le Chesnay, France) was then used to register the viable tumour tissue mask to ADC maps using a linear registration. This mask was also inverted to provide a non-viable tumour tissue mask.

\section{Multi-Gaussian model fitting}

Normalised histograms of ADC values from the entire tumour ROI in each patient (and at each time point where DWI was also performed prior to chemotherapy) were produced, with number of bins $=\log _{2}(N)+1$, where $N=$ number of voxels within the ROI [20]. To model subpopulations of ADC values within the tumour, a step-wise multi-Gaussian model fitting technique was used. This employed a non-linear least squares trust region algorithm [21] to fit the histogram to the following equation:

$$
y=\sum_{k=1}^{k_{\max }} a_{k} \cdot e^{\left[-\left(\frac{x-\mu_{k}}{c_{k}}\right)^{2}\right]}
$$

where $y=$ vector of normalised frequency values from the histogram, $k_{\max }=$ number of Gaussian components $\left(k_{\max }=\{1,2,3\}\right), a_{k}, \mu_{k}$ and $c_{k}=$ amplitude, mean and $\sqrt{2}{ }^{*}$ standard deviation of the $k$ th component Gaussian respectively, and $x=$ vector of ADC bin centres from the normalised histogram. Initially three Gaussian distributions were fit to the ADC histogram $\left(k_{\max }=3\right)$, using the 'fit' function in Matlab, which provided fitted values of the mean, standard deviation and amplitude of each distribution, as well as the $95 \%$ confidence interval $(\mathrm{Cl})$ for each fitted parameter. If the $\mathrm{Cl}$ surrounding the means of any of the distributions overlapped, the model was rejected. In this case, a two Gaussian model was used $\left(k_{\max }=2\right)$, and again, if the $\mathrm{Cl}$ of the two means overlapped, the process was repeated with a single Gaussian model $\left(k_{\max }=1\right)$.

Following model selection, the Mahalanobis distance [24] between the ADC value in each voxel within the tumour ROI and each of the fitted Gaussian components for that tumour was calculated (denoted $m_{k}$ for the Mahalanobis distance to the kth Gaussian component). For visualisation purposes, the Mahalanobis distance to each component Gaussian (ranging from $m_{1}$ for the distance to the lowest-ADC component, to $m_{3}$ for the distance to highestADC component, if $k_{\max }=3$ ) was normalised to the sum of all the Mahalanobis distances in that voxel (e.g. $m_{1 n}=m_{1} /\left(m_{1}+m_{2}+m_{3}\right)$ if $k_{\max }=3$, or $m_{1 n}=m_{1} /\left(m_{1}+m_{2}\right)$ if $\left.k_{\max }=2\right)$. For tumours in which $k_{\max }=3$, values of $1-m_{1 n}, 1-m_{2 n}$ and $1-m_{3 n}$ were assigned to the red, green and blue colour channels of the ADC image respectively, so that voxels closest to the lowest-ADC peak appeared red, voxels closest to the middle-ADC peak appeared green, and voxels closest to the highest-ADC peak appeared blue. Similarly if $k_{\max }=2$, just the red and green channels were used, with voxels closest to the lower-ADC peak appearing red, and voxels closest to the higher-ADC peak appearing green. This process is illustrated for an example patient in Figure 1. 


\section{Identification of non-viable tissue in ADC maps}

ADC values from voxels which were classified as containing either viable or non-viable tumour tissue using the viable/non-viable tissue masks (see 2.3 Post Processing) were pooled across the cohort. ROC analysis was then used to determine the optimum upper threshold for viable tumour tissue $A D C$ values $\left(A D C_{\text {viable }}\right)-A D C$ values above this were classified as representing non-viable tissue. This threshold value was then used in every patient to classify any ADC subpopulation centred above $A D C_{\text {viable }}$ as a 'non-viable' subpopulation.

\section{Differentiation of Wilms tumour subtypes}

ADC histograms from the post-chemotherapy scans were used for comparison with the histological assessment of the tumour, as these were most representative of the final excised tissue (the composition of the tumour tissue will generally change considerably during chemotherapy [2], [8], so pre-chemotherapy ADC data were not used in this part of the analysis). As histological quantification of stromal, blastemal and epithelial cells was only performed in viable tumour tissue, sub-populations from the multi-Gaussian model centred on an $A D C$ value greater than $A D C_{\text {viable }}$ were excluded. Following this, the sub-population within the ADC histogram with the greatest peak height was identified (referred to henceforth as $A D C_{1}$ ) - this represented the predominant viable tissue in the tumour, which is most representative of the tissue that is assessed histologically. The following summary parameters were evaluated for this sub-population: the mean $A D C$ value $\left(A_{1} C_{1 \text { MEAN }}\right.$ ), and the lower quartile $A D C$ value $\left(A D C_{1 L Q}\right)$. The lower quartile $A D C$ value was chosen to capture regions of high cellularity within the predominant sub-population, and is in accordance with several other studies investigating ADC histogram properties for differentiation of tumour grade [25], [26]. Note basing an analysis on the minimum ADC value is unreliable, as an entire tumour is classified based on the value in a single voxel.

For comparison, the same parameters from the 'raw' $A D C$ histogram $\left(A\right.$ C $_{\text {OMEAN }}$ and $A D C_{O L Q}$ ), representing the entire tumour volume without the multi-Gaussian model being applied, were also calculated. After confirming the data were normally distributed using Lilliefors test ( $5 \%$ significance level), a one-way ANOVA was used to test for significant differences in these summary parameters $\left(A D C_{1 M E A N}, A D C_{1 L Q}, A D C_{0 M E A N}\right.$ and $\left.A D C_{0 L Q}\right)$ across WT subtypes, followed by a Tukey-Kramer posthoc test to determine which subtypes were significantly different $(p<0.05)$.

\section{Results}

Mean patient age was 3.3 years (range 0.5-12.1 years), and the mean interval between preand post-chemotherapy scans was 46 days. None of the patients included in our cohort showed histological evidence of anaplasia.

Example histological sections demonstrating the various elements (blastemal, epithelial, stromal and regressive) found within a WT are shown in Figure 2. These different cell types can all be found within a single WT. Overall, using the multi-Gaussian model we found that prior to chemotherapy, $51 \%$ of ADC histograms were best fit using 2 Gaussian distributions, $29 \%$ were best fit with 3 Gaussians, and $20 \%$ were best fit with a single Gaussian. A similar pattern was observed post-chemotherapy, with $48 \%, 33 \%$ and $18 \%$ of tumours requiring for 2, 3, and 1 Gaussian distributions respectively. In the lesions for which DWI data were available both pre- and post-chemotherapy (23 in total), the change in the number of fitted Gaussian components as a result of chemotherapy was as follows: $-2: 4 \%,-1: 17 \%, 0: 38 \%$, $+1: 29 \%$ and $+2: 12 \%$. This indicates that in $38 \%$ of tumours, the number of ADC 
subpopulations with distinct cellular density within the tumour tissue did not change as a result of chemotherapy. The mean $r^{2}$ value for all multi-Gaussian fits was 0.97.

The methodology described in section 2.4 was tested against the established EM algorithm for fitting Gaussian mixture models [22], [23]. The EM algorithm gave very similar results, with a mean difference of only $4.3 \%$ per tumour in the fitted value of $A D C_{1 L Q}$ compared to our original analysis. However, the methodology presented here was found to be more stable, converging on exactly the same values over multiple iterations, whereas the EM algorithm was found to vary from one iteration to the next, with a mean variation of $2.5 \%$ in the calculated value of $A D C_{1 L Q}$ across 10 iterations. Unlike the standard implementation of the EM algorithm, the methodology presented here places strict limits on the degree of overlap between the component Gaussian distributions, which ensures the fitted Gaussian components represent subpopulations with distinct cellularity. We believe this also contributes to the stability of the fitting process, and therefore this methodology was better suited to this study.

\section{$A D C$ in viable/non-viable tissue}

The mean $( \pm$ SEM) ADC value in all viable tumour tissue voxels across the cohort was (1.54 $\pm 0.0014) \times 10^{-3} \mathrm{~mm}^{2} / \mathrm{s}$, compared to $(1.98 \pm 0.0025) \times 10^{-3} \mathrm{~mm}^{2} / \mathrm{s}$ in all necrotic tissue voxels (two tailed unpaired $t$-test, $\mathrm{p}<0.001$ ). An ROC analysis, illustrated in Figure 3 , determined the optimum upper threshold for viable tissue voxels to be $A D C_{\text {viable }}=1.81 \times 10^{-3} \mathrm{~mm}^{2} / \mathrm{s}(67 \%$ sensitivity, $75 \%$ specificity, area under the curve $=0.72$ ). An example of how this threshold can be used to identify sub-populations of non-viable tissue from the multi-Gaussian ADC model is shown in Figure 4. The green and blue coloured peaks in this example (Fig. 4E) were centred above $A D C_{\text {viable, }}$ and as such were identified as sub-populations of non-viable tissue (likely to be oedematous regions). This was further confirmed by the fact that these components of the ADC histogram were markedly reduced when the same tumour was analysed using the viable tissue mask (Fig. 4C). Furthermore, when comparing Fig. 4B and Fig. 4D it was clear that voxels belonging to these sub-populations (shown in green and blue in Fig. 4D) showed good visual correlation with the excluded regions from the viable tissue tumour mask (Fig. 4B), albeit with a slight discrepancy in places, which is discussed in section 4.1 .

\section{Assessment of treatment response}

Example ADC histograms, before and after treatment, are shown for a representative patient in Figure 5. In this example, both pre- and post-chemotherapy, the multi-Gaussian model identified three sub-populations within the tumour. Prior to chemotherapy, the majority of the tumour tissue was described by a prominent sub-population with a low ADC value $\left(A_{1 M C A N}=6.7 \times 10^{-4} \mathrm{~mm}^{2} / \mathrm{s}\right.$, red in Fig $\left.5 \mathrm{C}\right)$, representing highly cellular tissue, with another smaller peak in the $A D C$ histogram centred at a value greater than $A D C_{\text {viable }},\left(2.73 \times 10^{-3}\right.$ $\mathrm{mm}^{2} / \mathrm{s}$, blue in Fig $5 \mathrm{C}$ ), which is likely to represent oedematous, non-viable regions of the tumour. Between these peaks, a third subpopulation with a broad distribution of ADC values was observed, with a peak centred at $1.4 \times 10^{-3} \mathrm{~mm}^{2} / \mathrm{s}$, which is likely to represent voxels containing a mixture of viable and oedematous tissue. Following chemotherapy, the centre of this broad central peak shifted slightly to the right. The high-ADC peak remained in the same position, with approximately the same height. The low-ADC peak remained centred on approximately the same value, but showed a marked decrease in height, indicating that chemotherapy had significantly reduced the volume of viable tumour tissue in this patient, but the cellular density of the viable tissue that remained was unchanged. Note this result 
would be obscured if a single mean or median value for the entire ADC histogram were reported.

Overall, the 23 tumours in which DWI data were available both pre- and post-chemotherapy consisted of the following histological subtypes (number of tumours in brackets): stromal (6), epithelial (4), blastemal (4), mixed (7), and regressive (2). Ladder plots of ADC $_{1 \text { MEAN }}$ in these tumours, before and after chemotherapy, are shown in Figure 6. For each subtype, the mean $( \pm S D)$ intra-tumour change in $\mathrm{ADC}_{1 \mathrm{MEAN}}$ as a result of chemotherapy was: stromal: $+68 \pm 42 \%$ $(p=0.005$, paired $t$-test $)$, epithelial: $+26 \pm 24 \% \quad(p=0.12)$, blastemal: $+5 \pm 27 \% \quad(p=0.70)$, regressive: $+120 \pm 102 \%$ ( $p=N / A$, only 2 lesions), mixed: $+63 \pm 72 \% \quad(p=0.06)$. For each subtype, the mean $( \pm S D)$ intra-tumour change in volume as a result of chemotherapy (determined by multiplying the number of voxels within the tumour ROI by the voxel volume) was: stromal: $+15 \pm 61 \% \quad(p=0.68)$, epithelial: $-51 \pm 36 \% \quad(p=0.15)$, blastemal: $-62 \pm 29 \%$ $(p=0.25)$, regressive: $-54 \pm 56 \%(p=N / A$ (only 2 lesions $)$ ), mixed: $-27 \pm 47 \%(p=0.28)$.

\section{Differentiation of Wilms tumour subtypes}

The 32 tumours for which post-chemotherapy ADC data were available comprised the following histological subtypes (number of tumours in brackets): stromal (6), regressive (5), mixed (11), epithelial (4), blastemal (5), completely necrotic (1). As there was only one completely necrotic tumour, this subtype was excluded from this part of the analysis. The results from ANOVA tests of both the multi-Gaussian parameters $\left(\mathrm{ADC}_{1 \mathrm{MEAN}}, \mathrm{ADC}_{1 \mathrm{LQ}}\right)$ and raw histogram parameters $\left(A_{D C} C_{O M E A N}, A D C_{0 L Q}\right)$ across the WT subtypes are shown in Table 1. Both $A D C_{1 M E A N}$ and $A D C_{1 L Q}$ were more effective at separating WT subtypes than their counterparts from the raw ADC histogram, and indeed no significant difference was observed between any WT subtypes using the mean of the raw $A D C$ histogram ( $A D C_{\text {OMEAN }}$ ). The most significant difference between WT subtypes was found in the $A_{D C} C_{1 L Q}$ parameter (ANOVA $p=0.0004$ ), and a box and whisker plot for this parameter is shown in Figure 7. Blastemal tumours had significantly lower $A D C_{1 L Q}$ values than the stromal, regressive and mixed subtypes $(p<0.05)$, but there was no significant difference between blastemal and epithelial lesions $(p=0.35)$. An ROC analysis indicted that the optimum ADC $_{1 L Q}$ threshold value, in terms of differentiating epithelial and blastemal WTs (group 1) from the other subtypes (group 2), was $0.99 \times 10^{-3} \mathrm{~mm}^{2} / \mathrm{s}(91 \%$ sensitivity, $89 \%$ specificity, area under curve $=0.81-$ indicated by a horizontal line in Figure 7). Note, the tumour labeled ' $A$ ' in Figure 7, which was classified histologically as mixed, comprised $50 \%$ epithelial cells, $35 \%$ blastemal cells, and $15 \%$ stromal cells. So although this tumour was histologically referred to as mixed, the predominance of blastemal and stromal cells in the viable tissue explains the similarity between the $A D C_{1 L Q}$ value in this tumour with the other epithelial/blastemal tumours. Similarly, the outlier from the mixed group with the high value of $\mathrm{ADC}_{1 \mathrm{LQ}}$ (labeled ' $\mathrm{B}$ ' in Figure 7) was classified histologically as mixed, with predominant widespread stromal elements. This may explain why the $A D C_{1 L Q}$ value found in this lesion was more similar to the stromal group.

\section{Discussion}

Previous studies of chemotherapy response in WT have been largely based on histopathology, and as such it has not been possible to study the cellular composition of the same tumour at two time points. MRI provides a unique opportunity to measure morphological and functional changes in the same tumour over time. Conventionally, MRI has only been used to assess chemotherapy response by measuring the change in tumour volume. However, our data illustrate how chemotherapy-induced change in the cellularity of 
tumour tissue can also be assessed, which is not correlated with a change in tumour volume.

The most common subtype in our cohort was mixed (34\% of tumours), followed by stromal, blastemal and regressive tumours (19\%, 16\%, and 16\% respectively), epithelial $(12 \%)$ and completely necrotic (3\%). This shows a broadly similar pattern to the larger cohort studied by Weirich et al. [8], although in that study regressive was the most frequent subtype (after chemotherapy), and epithelial the least common. In Weirich et al.'s study, 71 patients who received immediate surgery without chemotherapy were also included, comprising $45 \%$ mixed, $39 \%$ blastemal and $16 \%$ epithelial WTs. The noticeable absence of stromal WTs in this group has been verified in other studies, which have reported very low incidences $(<2 \%)$ of stromal WTs in immediately operated patients [6], [27]. Weirich et al. suggest that chemotherapy induces further differentiation and maturation of other subtypes into stromal predominant WTs. In our study, we were able to monitor the change in ADC (and therefore cellular density) as a result of chemotherapy in tumours classified as stromal after treatment. Our data support this hypothesis, since every stromal tumour in our cohort underwent a marked increase in ADC in the viable tissue as a result of chemotherapy. This suggests all these tumours were composed originally of tissue with a higher cellularity, such as immature blastema or stroma, which decreased significantly after chemotherapy, with the positive shift in ADC reflecting maturation into mature stromal tissue. Interestingly, this was not reflected in the change in tumour volume - despite a fairly uniform increase in ADC, 2 stromal tumours showed a marked increase in volume, 2 showed a very slight increase, and 2 showed marked shrinkage as a result of chemotherapy.

It has been suggested that blastema is the most responsive tumour component to chemotherapy [28], however WTs which remain predominantly blastemal after pre-operative chemotherapy are most likely to develop relapse [8]. We found that $A D C_{1 L Q}$ was the best parameter for identifying blastemal-type WTs, with these having the lowest ADC $_{1 L Q}$ values, suggesting the highest cellularity of all the subtypes. Of all the subtypes, our blastemal WTs also showed the greatest shrinkage in volume as a result of chemotherapy (mean $-62 \%$ volume change), as well as the smallest chemotherapy-induced change in ADC $_{1 \text { MEAN }}$ in the remaining viable tissue (mean 5\% increase). This would confirm the hypothesis that blastemal cells are the most responsive to chemotherapy, represented by the high level of tumour shrinkage, but the viable tissue that remains following chemotherapy is chemoresistant, retaining a very low ADC value (high cellularity).

All the epithelial WTs in our cohort showed a positive shift in ADC $_{1 \text { MEAN }}$ as a result of chemotherapy, along with a shrinkage in volume. However, the shift in ADC $_{1 \text { MEAN }}$ was small (only blastemal WTs showed a smaller increase), so, similar to blastemal WTs, chemotherapy was effective at shrinking epithelial WTs, but the remaining viable tissue was highly cellular.

Although we only had pre- and post-chemotherapy DWI data for 2 regressive WTs, which precludes a statistical analysis of treatment response in this subtype, both of these regressive tumours showed a marked increase in $A C_{1 M E A N}$ as a result of chemotherapy, along with a small amount of tumour shrinkage. Regressive WTs often include regions of hypo-cellular tissue containing foamy and/or haemosiderin-laden macrophages [29]. The appearance of these hypo-cellular regions after chemotherapy is likely to be a major contributing factor to the large increase in $\mathrm{ADC}_{1 \text { MEAN }}$ values seen in these lesions. 


\section{Limitations}

The limited size of our cohort precludes a rigorous statistical analysis of chemotherapy response for each WT subtype. However, the primary aim of this study was to introduce the multi-Gaussian modelling approach to ADC histogram analysis, demonstrate its potential to identify the most prominent viable tissue sub-population in the tumour, and highlight the effect on the ADC values in this sub-population in high-risk WTs. Also, although our ADC viable $_{\text {e }}$ threshold appears effective at removing non-viable oedematous tissue, ADC values in necrotic areas can occasionally be very low, mimicking highly cellular tumour tissue [2], and these will not be excluded in our analysis. Similarly, the slight discrepancy between the T1based viable tissue masks and the ADC maps, which tends to occur in regions that have high-ADC but are included within the viable tissue mask, could be due to partial volume artefacts in the DWI data. The T1-weighted data were acquired at a higher spatial resolution than the DWI data, and will be less susceptible to partial volume effects in voxels surrounding oedematous regions, hence non-viable regions on the viable tissue mask occasionally appear smaller in size. Furthermore, the threshold for viable tissue signal on the subtracted T1w images, based on the signal in the erector spinae muscles, may need further refinement, as occasionally voxels which show only marginal levels of enhancement on postGadolinium T1w images may be incorrectly classified as viable tissue.

Due to the rarity of anaplasia in WT (approx. 5\% of patients), none of the patients in our cohort showed evidence of anaplasia on histological assessment, so the effect of this on ADC values could not be assessed. Also, although in-plane partial volume artefacts were minimised by only including tumours with an area greater than $3 \mathrm{~cm}^{2}$, we were unable to account for through-plane partial volume artefacts. Lastly, while $A D C_{1\llcorner Q}$ was effective at separating blastemal WTs from stromal, regressive and mixed subtypes, it could not separate blastemal and epithelial tumours. Both these subtypes contain highly cellular tissue, but only blastemal tumours are classified as 'high-risk'. Further work is needed to separate these subtypes in vivo, perhaps using a larger cohort, combined with novel methods to register the source location of histology tissue samples onto ADC maps. Perfusion-weighted imaging may also help to differentiate these WT subtypes.

\section{Conclusion}

The ADC values provided by DWI provide a surrogate biomarker of cellular density, however, WTs are heterogeneous neoplasms, and it is clear that distinct cellular subpopulations usually exist within a single tumour. Therefore, an overall mean or percentile $A D C$ value obtained for the entire tumour will not give an accurate representation of tumour composition, as high ADC values in non-viable tissue regions will counteract low ADC values in highly cellular regions. Furthermore, distinct cellular regions within a tumour may respond differently to chemotherapy, so treatment response cannot be assessed reliably using summary ADC values for the entire tumour. The multi-Gaussian model presented here provides an automated means for representing a WT based on sub-populations with distinct cellularities, and identifying the predominant viable tissue sub-population, which will be closest matched to the tissue assessed by histological analysis. Using the multi-Gaussian model, the mean and lower quartile ADC values from the predominant viable sub-population are demonstrated to be better at differentiating histologically determined WT subtypes than the same metrics from the entire tumour, and can be used to separate highly cellular epithelial or blastemal predominant regions, from less cellular stromal, regressive and mixed regions. This information can be used to stratify patients based on the likely predominant cell type in a WT at an early stage, and could help surgeons target initial needle biopsies on more malignant tissue. Identifying regions which are more likely to contain malignant cells would also be helpful when planning nephron-sparing surgery, particularly in patients with bilateral WTs. 
Lastly, there is emerging evidence that the current definition of 'blatemal-type' WT is somewhat subjective, as it does not measure the total volume of blastemal cells which survive chemotherapy, but rather the percentage of blastemal cells in the viable tissue. As such, a large WT classified as intermediate-risk may contain a greater absolute volume of blastemal cells than a smaller high-risk blastemal-type WT. A recent analysis has shown that there is a threshold for the total volume of blastemal cells that survive preoperative chemotherapy, of between 20-50 ml, that confers adverse event free survival, regardless of the histological classification of the WT [30]. However, total blastemal tissue volume is challenging to measure with histological techniques. By combining histological analysis of tumour tissue, which would remove the ambiguity between the predominance of blastemal / epithelial tissue, with an estimation of the total volume of low ADC tissue from the multiGaussian model, an estimate of the total blastemal volume could be made. This would provide a new biomarker which would further refine the current clinical definition of 'high-risk' histology, and could be used to guide post-operative management of future WT patients.

\section{References}

[1] G. Pastore, A. Znaor, F. Spreafico, N. Graf, K. Pritchard-Jones, and E. SteliarovaFoucher, "Malignant renal tumours incidence and survival in European children (19781997): report from the Automated Childhood Cancer Information System project," European journal of cancer, vol. 42, no. 13, pp. 2103-2114, Sep. 2006.

[2] K. McDonald, N. J. Sebire, J. Anderson, and O. E. Olsen, "Patterns of shift in ADC distributions in abdominal tumours during chemotherapy-feasibility study," Pediatric Radiology, vol. 41, no. 1, pp. 99-106, Jan. 2011.

[3] K. W. Gow, I. F. Roberts, D. H. Jamieson, H. Bray, J. F. Magee, and J. J. Murphy, "Local staging of Wilms' tumor--computerized tomography correlation with histological findings," Journal of pediatric surgery, vol. 35, no. 5, pp. 677-679, May 2000.

[4] Ø. E. Olsen, A. C. Jeanes, N. J. Sebire, D. J. Roebuck, A. J. Michalski, R. A. Risdon, and C. M. Owens, "Changes in computed tomography features following preoperative chemotherapy for nephroblastoma: relation to histopathological classification," European radiology, vol. 14, no. 6, pp. 990-994, Jun. 2004.

[5] D. M. Green, J. B. Beckwith, N. E. Breslow, P. Faria, J. Moksness, J. Z. Finklestein, P. Grundy, P. R. Thomas, T. Kim, and S. Shochat, "Treatment of children with stages II to IV anaplastic Wilms' tumor: a report from the National Wilms' Tumor Study Group," Journal of Clinical Oncology: Official Journal of the American Society of Clinical Oncology, vol. 12, no. 10, pp. 2126-2131, Oct. 1994.

[6] J. B. Beckwith, C. E. Zuppan, N. G. Browning, J. Moksness, and N. E. Breslow, "Histological analysis of aggressiveness and responsiveness in Wilms' tumor," Medical and pediatric oncology, vol. 27, no. 5, pp. 422-428, Nov. 1996.

[7] G. M. Vujanić, B. Sandstedt, D. Harms, A. Kelsey, I. Leuschner, J. de Kraker, and SIOP Nephroblastoma Scientific Committee, "Revised International Society of Paediatric Oncology (SIOP) working classification of renal tumors of childhood," Medical and pediatric oncology, vol. 38, no. 2, pp. 79-82, Feb. 2002.

[8] A. Weirich, I. Leuschner, D. Harms, G. M. Vujanic, J. Tröger, U. Abel, N. Graf, D. Schmidt, R. Ludwig, and P. A. Voûte, "Clinical impact of histologic subtypes in localized non-anaplastic nephroblastoma treated according to the trial and study SIOP-9/GPOH," Annals of Oncology, vol. 12, no. 3, pp. 311-319, Mar. 2001.

[9] J. de Kraker, N. Graf, H. van Tinteren, F. Pein, B. Sandstedt, J. Godzinski, M. F. Tournade, and SIOP, "Reduction of postoperative chemotherapy in children with stage I intermediate-risk and anaplastic Wilms' tumour (SIOP 93-01 trial): a randomised controlled trial," Lancet, vol. 364, no. 9441, pp. 1229-1235, Oct. 2004.

[10] N. Graf, H. van Tinteren, C. Bergeron, F. Pein, M. M. van den Heuvel-Eibrink, B. Sandstedt, J.-P. Schenk, J. Godzinski, F. Oldenburger, R. Furtwängler, and J. de 
Kraker, "Characteristics and outcome of stage II and III non-anaplastic Wilms' tumour treated according to the SIOP trial and study 93-01," European Journal of Cancer, vol. 48, no. 17, pp. 3240-3248, Nov. 2012.

[11] A. M. Termuhlen, J. M. Tersak, Q. Liu, Y. Yasui, M. Stovall, R. Weathers, M. Deutsch, C. A. Sklar, K. C. Oeffinger, G. Armstrong, L. L. Robison, and D. M. Green, "Twentyfive year follow-up of childhood Wilms tumor: a report from the Childhood Cancer Survivor Study," Pediatric blood \& cancer, vol. 57, no. 7, pp. 1210-1216, Dec. 2011.

[12] A. M. Smets and J. de Kraker, "Malignant tumours of the kidney: imaging strategy," Pediatric Radiology, vol. 40, no. 6, pp. 1010-1018, Jun. 2010.

[13] J. S. Dome, C. A. Cotton, E. J. Perlman, N. E. Breslow, J. A. Kalapurakal, M. L. Ritchey, P. E. Grundy, M. Malogolowkin, J. B. Beckwith, R. C. Shamberger, G. M. Haase, M. J. Coppes, P. Coccia, M. Kletzel, R. M. Weetman, M. Donaldson, R. M. Macklis, and D. M. Green, "Treatment of Anaplastic Histology Wilms' Tumor: Results From the Fifth National Wilms' Tumor Study," Journal of Clinical Oncology, vol. 24, no. 15, pp. 23522358, May 2006.

[14] P. D. Humphries, N. J. Sebire, M. J. Siegel, and $\varnothing$. E. Olsen, "Tumors in Pediatric Patients at Diffusion-weighted MR Imaging: Apparent Diffusion Coefficient and Tumor Cellularity," Radiology, vol. 245, no. 3, pp. 848 -854, Dec. 2007.

[15] F. P. A. Pereira, G. Martins, and R. de V. Carvalhaes de Oliveira, "Diffusion magnetic resonance imaging of the breast," Magnetic resonance imaging clinics of North America, vol. 19, no. 1, pp. 95-110, Feb. 2011.

[16] T. Parikh, S. J. Drew, V. S. Lee, S. Wong, E. M. Hecht, J. S. Babb, and B. Taouli, "Focal liver lesion detection and characterization with diffusion-weighted MR imaging: comparison with standard breath-hold T2-weighted imaging," Radiology, vol. 246, no. 3, pp. 812-822, Mar. 2008.

[17] C. K. Kim, B. K. Park, J. J. Han, T. W. Kang, and H. M. Lee, "Diffusion-weighted imaging of the prostate at $3 \mathrm{~T}$ for differentiation of malignant and benign tissue in transition and peripheral zones: preliminary results," Journal of computer assisted tomography, vol. 31, no. 3, pp. 449-454, Jun. 2007.

[18] B. Taouli, R. K. Thakur, L. Mannelli, J. S. Babb, S. Kim, E. M. Hecht, V. S. Lee, and G. M. Israel, "Renal Lesions: Characterization with Diffusion-weighted Imaging versus Contrast-enhanced MR Imaging," Radiology, vol. 251, no. 2, pp. 398-407, May 2009.

[19] B. Paudyal, P. Paudyal, Y. Tsushima, N. Oriuchi, M. Amanuma, M. Miyazaki, A. Taketomi-Takahashi, Y. Nakazato, and K. Endo, "The role of the ADC value in the characterisation of renal carcinoma by diffusion-weighted MRI," The British Journal of Radiology, vol. 83, no. 988, pp. 336-343, Apr. 2010.

[20] H. A. Sturges, "The Choice of a Class Interval," Journal of the American Statistical Association, vol. 21, no. 153, pp. 65-66, 1926.

[21] J. Moré and D. Sorensen, "Computing a Trust Region Step," SIAM Journal on Scientific and Statistical Computing, vol. 4, no. 3, pp. 553-572, Sep. 1983.

[22] A. P. Dempster, N. M. Laird, and D. B. Rubin, "Maximum likelihood from incomplete data via the EM algorithm," JOURNAL OF THE ROYAL STATISTICAL SOCIETY, SERIES B, vol. 39, no. 1, pp. 1-38, 1977.

[23] D. Simon, K. H. Fritzsche, C. Thieke, J. Klein, P. Parzer, M.-A. Weber, and B. Stieltjes, "Diffusion-weighted imaging-based probabilistic segmentation of high- and lowproliferative areas in high-grade gliomas," Cancer Imaging: The Official Publication of the International Cancer Imaging Society, vol. 12, pp. 89-99, 2012.

[24] P. Mahalanobis, "On the generalised distance in statistics," presented at the Proceedings National Institute of Science, India, 1936, vol. 2, pp. 49-55.

[25] T. Kobus, P. C. Vos, T. Hambrock, M. De Rooij, C. A. Hulsbergen-Van de Kaa, J. O. Barentsz, A. Heerschap, and T. W. J. Scheenen, "Prostate Cancer Aggressiveness: In Vivo Assessment of MR Spectroscopy and Diffusion-weighted Imaging at 3 T," Radiology, vol. 265, no. 2, pp. 457-467, Nov. 2012.

[26] R. F. Barajas Jr, J. L. Rubenstein, J. S. Chang, J. Hwang, and S. Cha, "Diffusionweighted MR imaging derived apparent diffusion coefficient is predictive of clinical 
outcome in primary central nervous system lymphoma," AJNR. American journal of neuroradiology, vol. 31, no. 1, pp. 60-66, Jan. 2010.

[27] P. Gutjahr, P. Kaatsch, H. Spaar, D. Niethammer, U. Göbel, G. Henze, R. Ludwig, J. Kühl, R. Erttmann, D. Harms, and R. Hohenfellner, "Klinik, Therapie und Prognose bei 373 Kindern mit Wilms-Tumoren - Ergebnisse der bundesweiten Studie 1980-1988," Aktuelle Urologie, vol. 21, no. 03, pp. 132-141, May 1990. English translation of abstract available at: https://www.thiemeconnect.com/products/ejournals/abstract $/ 10.1055 / \mathrm{s}-2008-1060616$ ?device $=$ mobile

[28] C. W. Zuppan, J. B. Beckwith, D. A. Weeks, D. W. Luckey, and K. C. Pringle, "The effect of preoperative therapy on the histologic features of Wilms' tumor. An analysis of cases from the Third National Wilms' Tumor Study," Cancer, vol. 68, no. 2, pp. 385394, Jul. 1991.

[29] G. M. Vujanić and B. Sandstedt, "The pathology of Wilms' tumour (nephroblastoma): the International Society of Paediatric Oncology approach," Journal of Clinical Pathology, vol. 63, no. 2, pp. 102-109, Feb. 2010.

[30] J. Dome, E. Periman, and N. Graf, Risk Stratification for Wilms Tumor: Current Approach and Future Directions. American Society of Clinical Oncology Educational Book, 2014. 


\section{Tables}

Table 1

Results from ANOVA tests of multi-Gaussian and raw histogram parameters across Wilms' tumour subtypes.

$p$-value $\quad$ Significant differences $(p<0.05$, Tukey-Kramer $)$

(ANOVA)

\section{Multi-Gaussian}

analysis

$\begin{array}{lll}\mathrm{ADC}_{1 \mathrm{MEAN}} & 0.0010 \quad \begin{array}{l}\text { blastemal vs. stromal, blastemal vs. regressive, } \\ \text { blastemal vs. mixed, epithelial vs. stromal }\end{array} \\ \mathrm{ADC}_{1 \mathrm{LQ}} & \begin{array}{l}0.00039 \\ \text { blastemal vs. stromal, blastemal vs. regressive, } \\ \text { blastemal vs. mixed, epithelial vs. stromal }\end{array}\end{array}$

Raw histogram

$\begin{array}{lcl}A C_{O M E A N} & 0.056 & \text { None } \\ \text { ADC }_{0 L Q} & 0.0049 & \text { blastemal vs. stromal, epithelial vs. stromal }\end{array}$




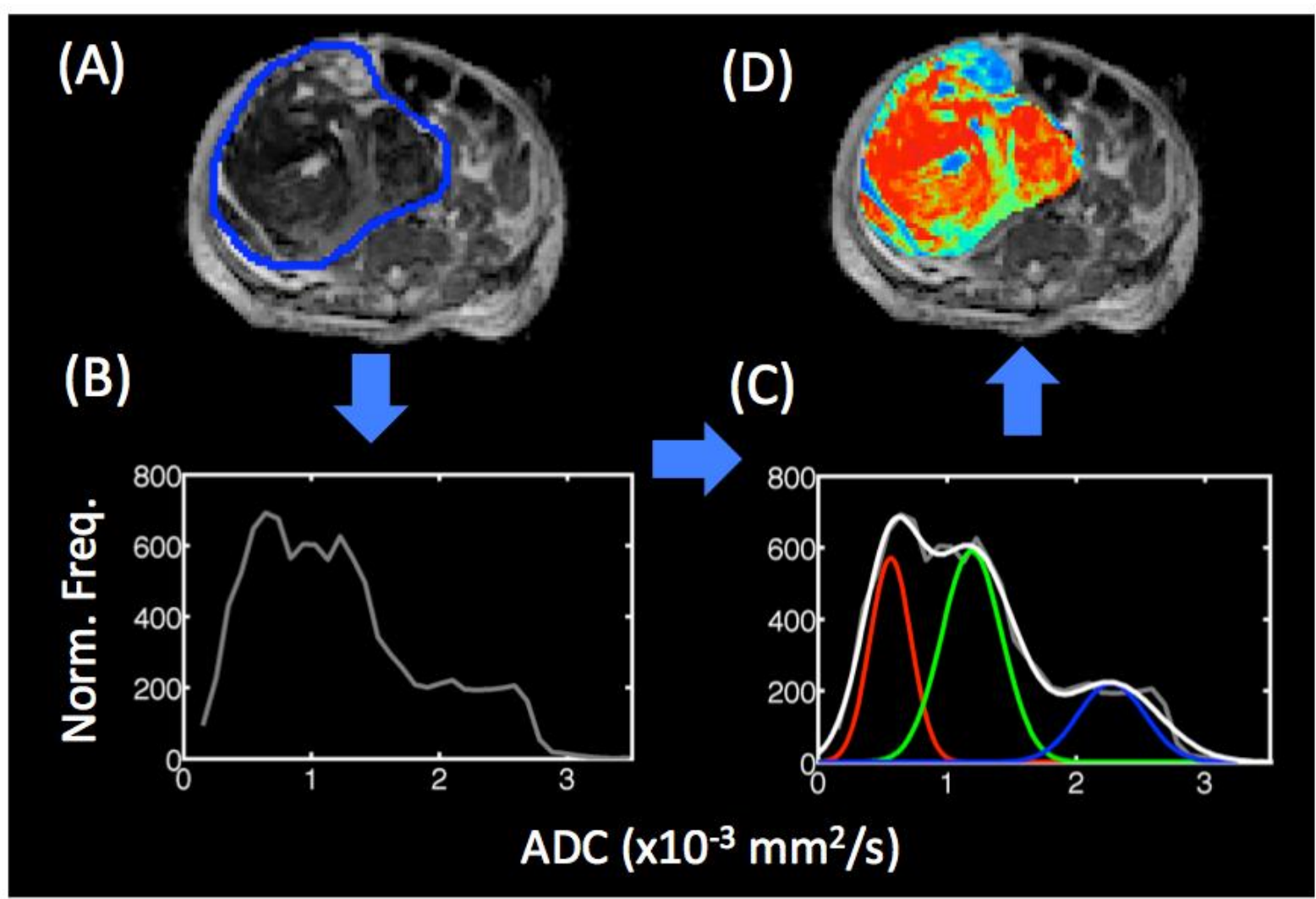

\section{Figure 1}

(A) ADC map, with the Wilms tumour ROI outlined in blue, and (B) the 'raw' ADC histogram from this tumour. (C) The multi-Gaussian model provides a fitted curve to the histogram (white line), in this case comprising three underlying Gaussian distributions (shown in red, green and blue). The Mahalanobis distance $(m)$ between the local ADC value and the three Gaussian components is calculated in each voxel in the tumour ROI, and normalised to the sum of all Mahalanobis distances in that voxel $\left(m_{n}\right)$. The red, green and blue channels of each tumour voxel in (D) represent $1-m_{n}$ for the three Gaussian components shown in $(C)$, with voxels closest to the lowest-ADC peak shown as red, those closest to the middle-ADC peak shown as green, and those closest to the highest-ADC peak shown as blue. 

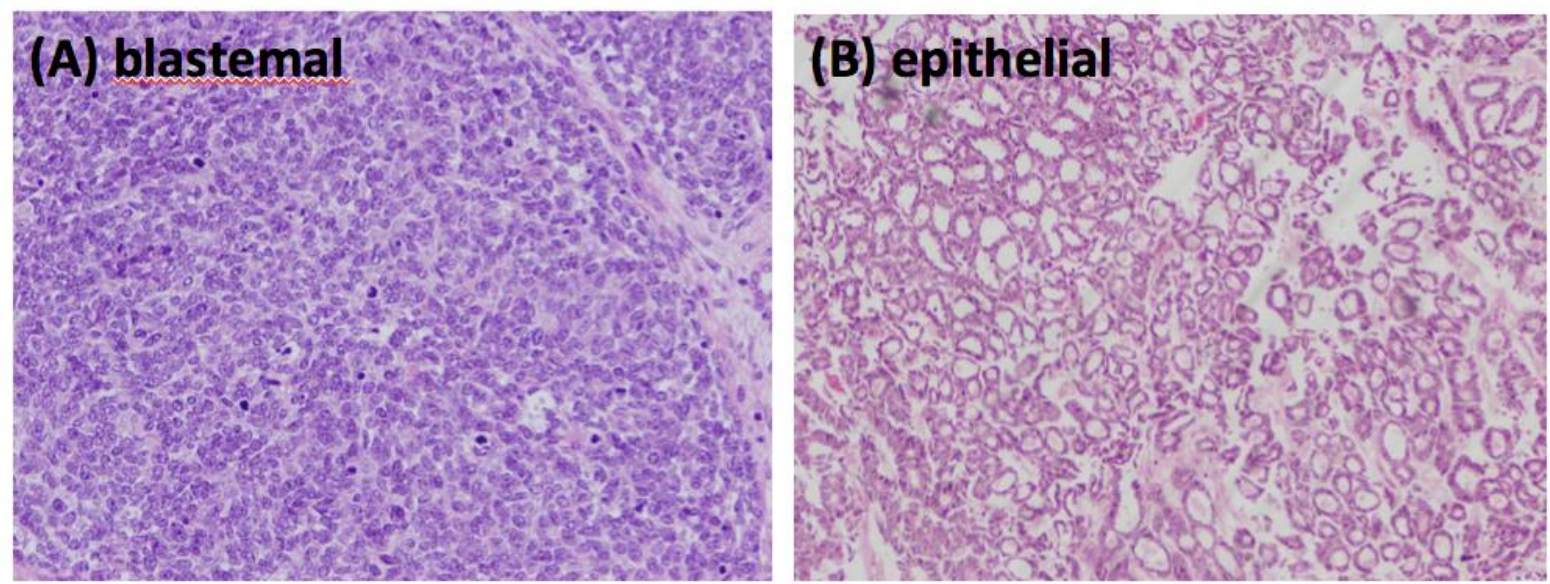

\section{(C) stromal}

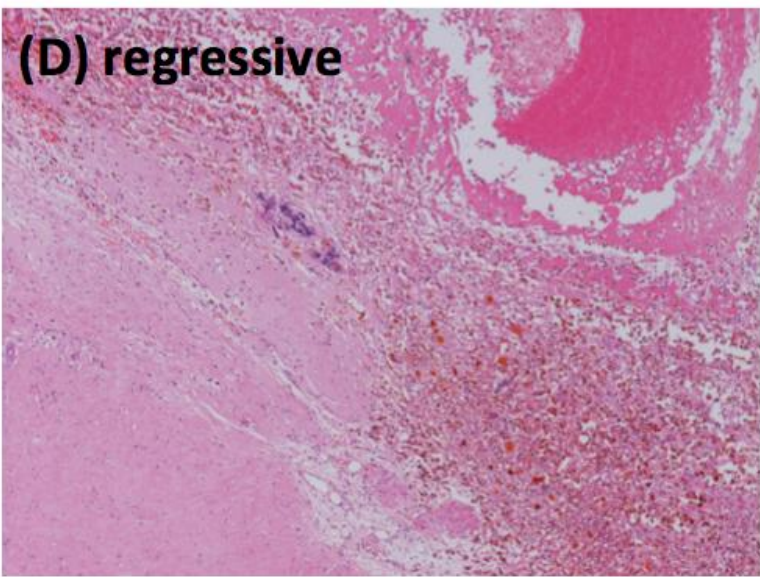

\section{Figure 2}

Histological sections demonstrating (A) blastemal, (B) epithelial, (C) stromal and (D) regressive tissue types within a Wilms' tumour (haematoxylin and eosin stain, original magnifications $\mathrm{x} 100, \mathrm{x} 100, \mathrm{x} 100$ and $\mathrm{x} 40$ respectively). 
(A)

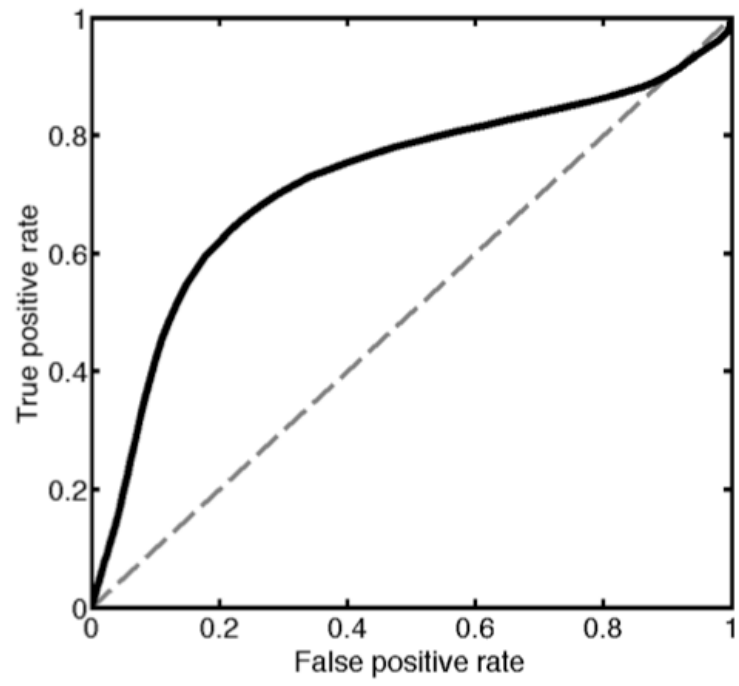

(B)

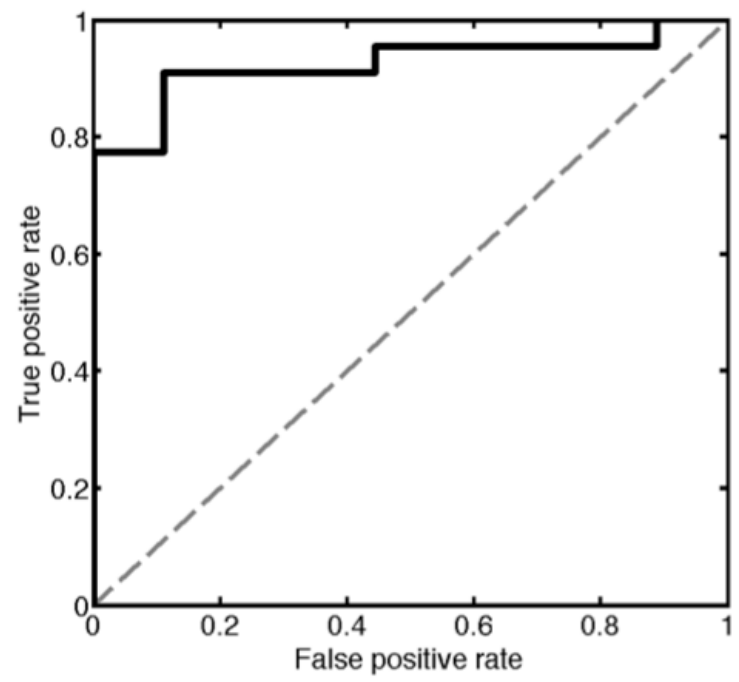

Figure 3

ROC curves, illustrating: $(A)$ the optimum $A D C_{\text {viable }}$ threshold value, for differentiating viable (group 1) and non-viable (group 2) tissue, and (B) the optimum $A \mathrm{AC}_{1 \mathrm{LQ}}$ threshold value, for differentiating epithelial and blastemal WTs (group 1) from the other subtypes (group 2). 


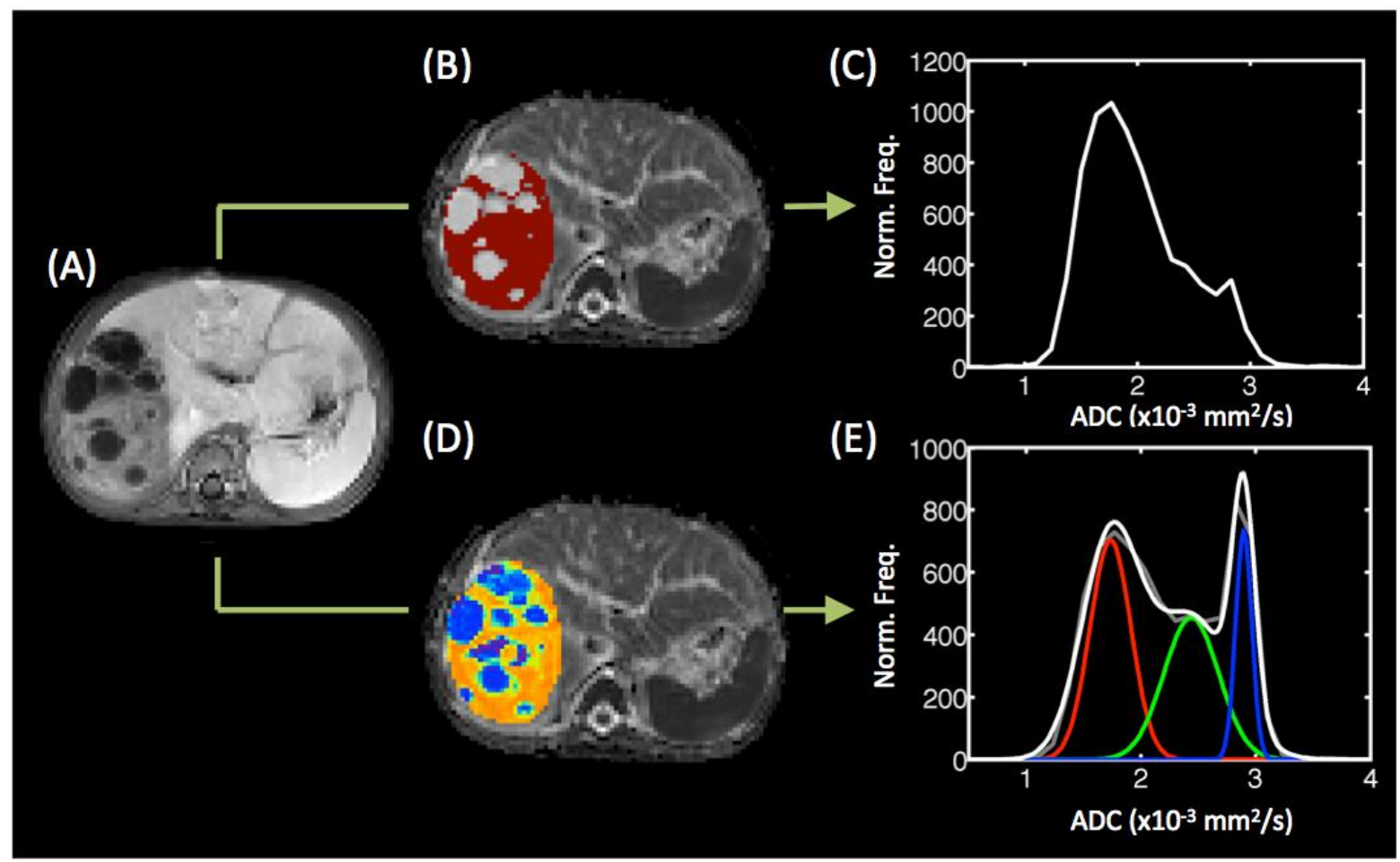

Figure 4

(A) T1w difference map (registered to DWI space), created by subtracting the pre-gadolinium signal intensity from the post-gadolinium signal, in a representative patient. The viable tumour tissue mask is shown in red in (B), and the ADC histogram for all voxels within this mask is shown in $(C)$. The mapping of the sub-populations from the multi-Gaussian model is shown in (D), along with the ADC histogram and 3 underlying sub-populations in (E). 


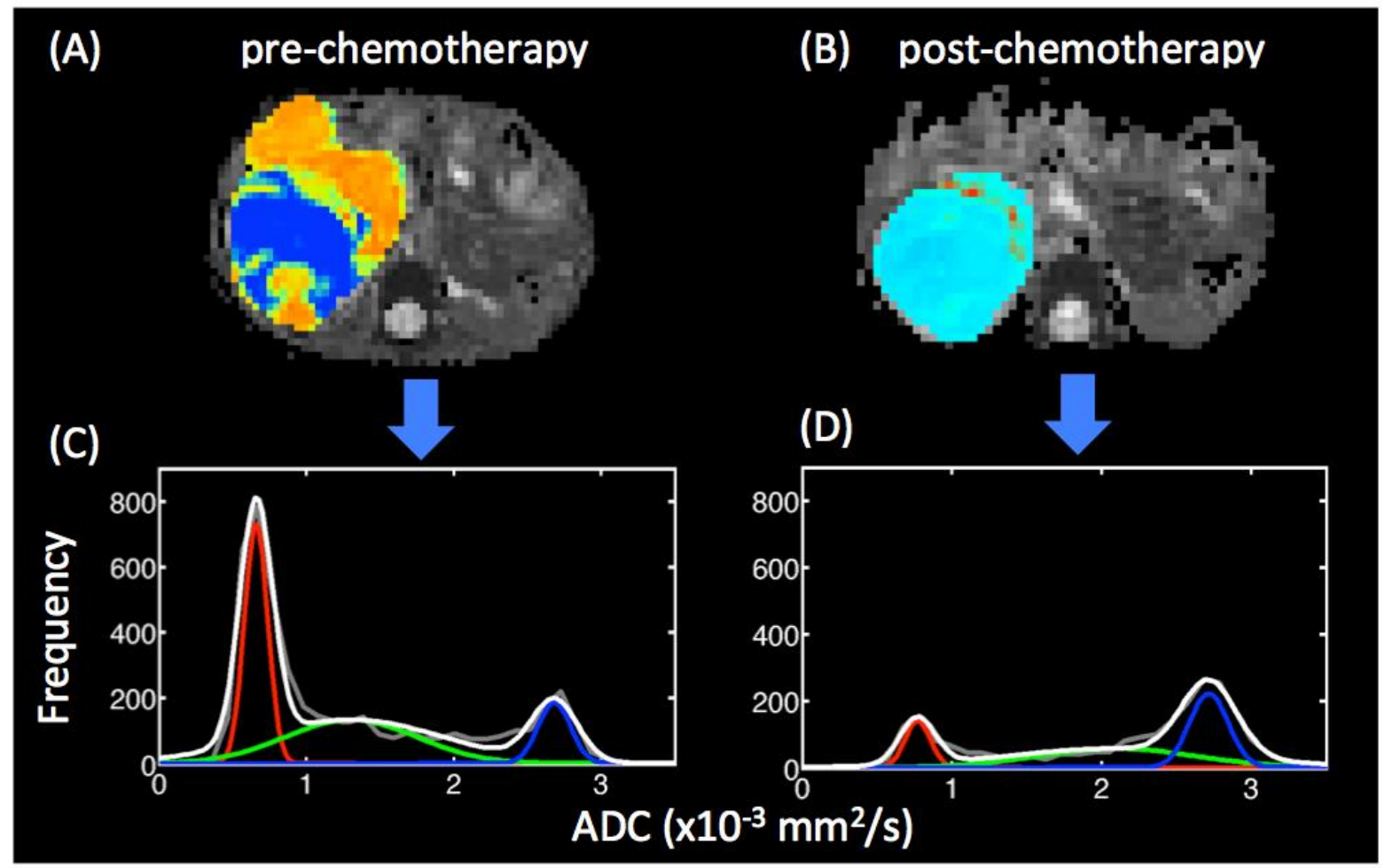

\section{Figure 5}

ADC maps for a blastemal Wilms' tumour patient pre- and post-chemotherapy are shown in (A) and (B) respectively, with voxels within the ROI coloured according to the Mahalanobis distance from each subpopulation (see Figure 1 for details). The corresponding ADC histograms are shown in (C) and (D) respectively, with the fit from the multi-Gaussian model overlaid. 


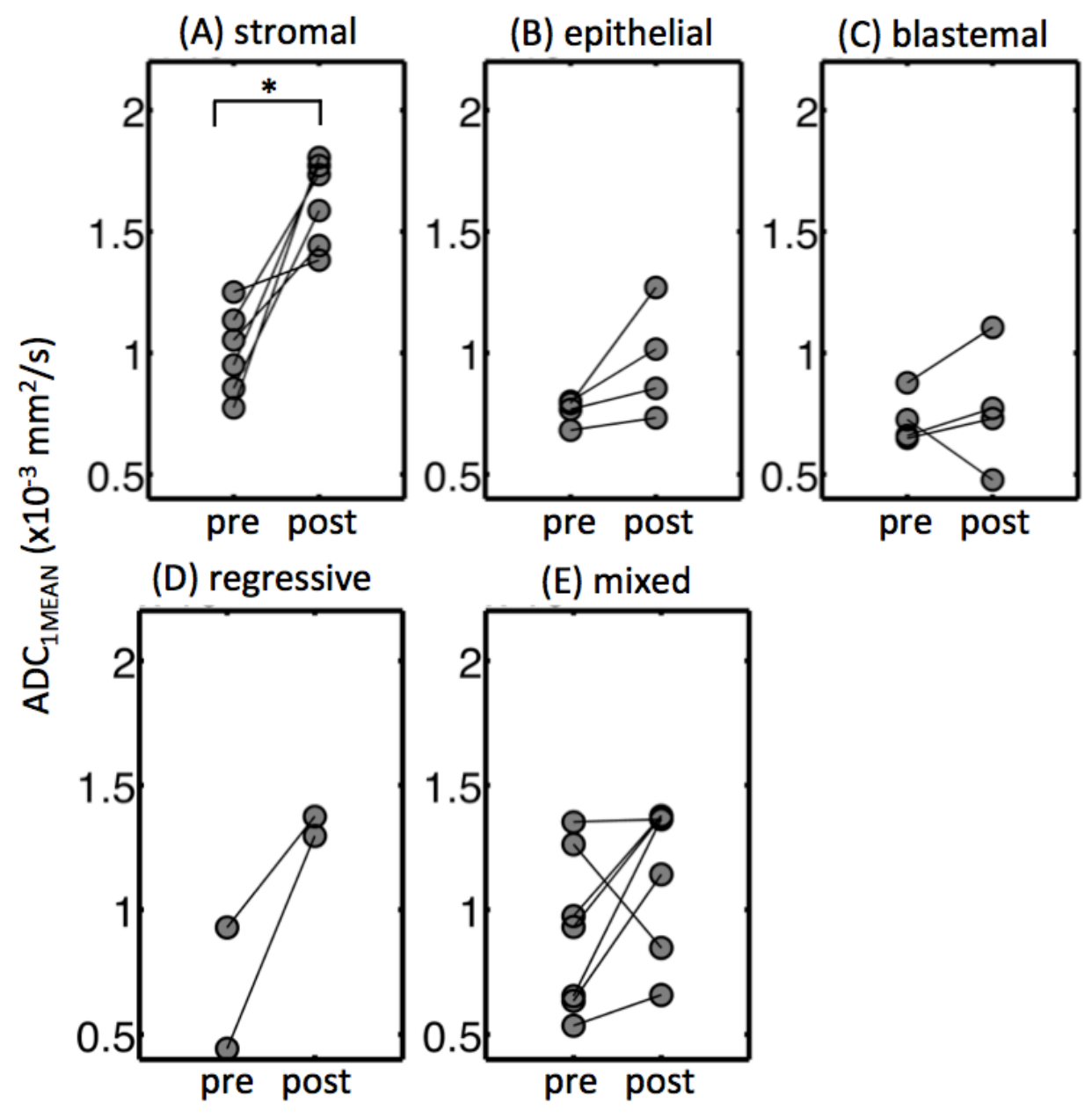

Figure 6

Ladder plots of $\mathrm{ADC}_{1 \mathrm{MEAN}}$ in different Wilms' tumour subtypes, with connected points representing the same tumour pre- and post-chemotherapy. A significant difference within each subtype between pre- and post-chemotherapy ADC $_{1 \text { MEAN }}$, determined using a twotailed paired $t$ test, is indicated by * $(p<0.05)$. No $t$ test was performed on the regressive group (D) due to the small number of lesions in this group. 


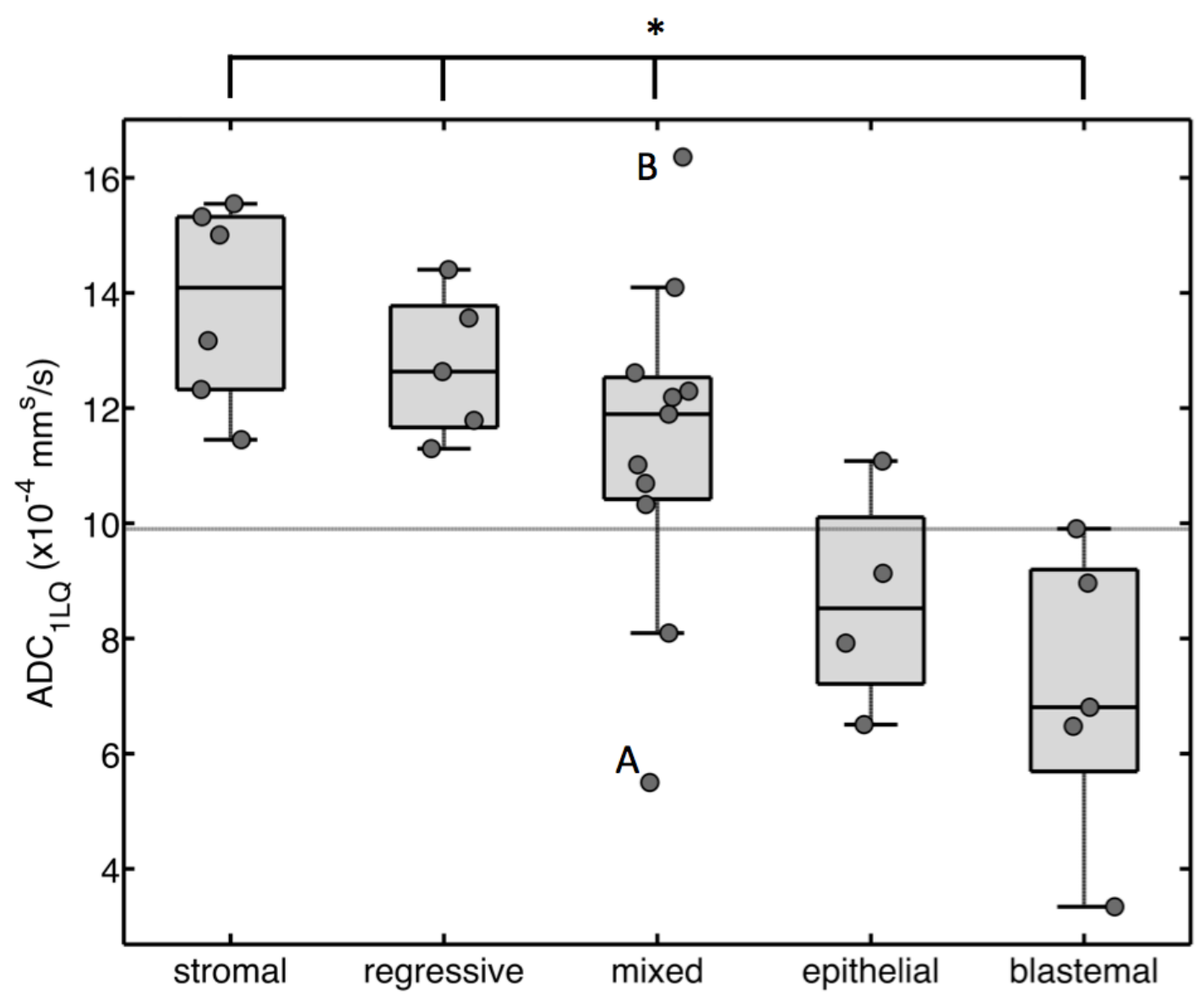

Figure 7

Box and whisker plot of $A D C_{1 L Q}$ across Wilms' tumour subtypes (post chemotherapy), with data points for individual tumours overlaid. Significant difference between blastemal tumours and all other subtypes, determined using an ANOVA / Tukey-Kramer posthoc test, is indicated by * $(p<0.05)$. There was no significant difference between the epithelial and blastemal subtypes. The optimum $\mathrm{ADC}_{1 \mathrm{LQ}}$ threshold value, in terms of differentiating epithelial and blastemal WTs (group 1) from the other subtypes (group 2), is overlaid (horizontal grey line at $A D C_{1 L Q}=0.99 \times 10^{-3} \mathrm{~mm}^{2} / \mathrm{s}$ ). The viable tissue in the tumour labelled ' $A$ ' was determined histologically to consist of $50 \%$ epithelial cells, $35 \%$ blastemal cells, and $15 \%$ stromal cells. The tumour labelled 'B' was determined histologically to consist of widespread stromal elements. 